\title{
Left atrial strain in the assessment of diastolic function: providing new insights into primary myocardial dysfunction in Marfan syndrome
}

Eusebio García-Izquierdo ( $\sim$ usegij@gmail.com )

Hospital Universitario Puerta de Hierro Majadahonda

Vanessa Moñivas-Palomero

Hospital Universitario Puerta de Hierro Majadahonda

Alberto Forteza

Hospital Universitario Puerta de Hierro Majadahonda

Carlos Martín-López

Hospital Universitario Puerta de Hierro Majadahonda

Mario Torres-Sanabria

Hospital Universitario Puerta de Hierro Majadahonda

Xabier Cia-Mendioroz

Hospital Universitario Puerta de Hierro Majadahonda

Consuelo Olivo-Rodríguez

Hospital Universitario Puerta de Hierro Majadahonda

Sara Navarro-Rico

Hospital Universitario Puerta de Hierro Majadahonda

Andrés Sánchez-Gómez

Hospital Universitario Puerta de Hierro Majadahonda

Jesús G. Mirelis

Hospital Universitario Puerta de Hierro Majadahonda

Miguel A. Cavero

Hospital Universitario Puerta de Hierro Majadahonda

Susana Mingo-Santos

Hospital Universitario Puerta de Hierro Majadahonda

Research Article

Keywords: Marfan syndrome, echocardiography, diastolic function, left atrium, strain

Posted Date: March 17th, 2021

DOI: https://doi.org/10.21203/rs.3.rs-300458/v1 
License: (9) (i) This work is licensed under a Creative Commons Attribution 4.0 International License. Read Full License

Version of Record: A version of this preprint was published at The International Journal of Cardiovascular Imaging on April 19th, 2021. See the published version at https://doi.org/10.1007/s10554-021-02247-7. 


\section{Abstract}

Previous studies using conventional echocardiographic measurements have reported subclinical left ventricular (LV) diastolic abnormalities in patients with Marfan syndrome (MFS). Left atrial (LA) strain allows an accurate categorization of LV diastolic dysfunction. We aimed to characterize LV myocardial performance in a cohort of MFS patients using STE-derived measurements (LV and LA strain) along with conventional echocardiographic parameters. We studied 127 adult patients with MFS (no prior cardiac surgery or significant valvular regurgitation) and 38 healthy controls. We performed detailed echocardiograms and selected left atrial reservoir strain (LASr) as a surrogate of impaired relaxation. Additionally, we searched for possible determinants of LASr in patients with MFS, with a special focus on the elastic properties of the aorta. In spite of lower E-wave, septal and lateral e' velocities and average E/e' ratio in MFS patients, all participants had normal diastolic function according to current guidelines. MFS patients exhibited reduced LV global longitudinal strain $(19.3 \pm 2.6$ vs $21.6 \pm 2.1 \%, p<0,001)$ and reduced $\operatorname{LASr}(32.9 \pm 8.5$ vs $43.3 \pm 7.8 \%, p<0.001)$ compared to controls. In the MFS cohort, we found weak significant $(p<0.05)$ correlations between LASr and certain parameters: $E / A$ ratio $(R=0.258)$, $E$ wave $(R=0.226)$, aortic distensibility $(R=0.222)$, stiffness index $(R=-0.216), L V$ ejection fraction $(R=0.214)$, lateral $e^{\prime}(R=0.210)$, $L V$ end-systolic volume index $(R=-0.210), L V$ global longitudinal strain $(R=0.201)$, septal e' $(R=0.185)$. After multivariate analysis, only LV end-systolic volume index and $E / A$ ratio maintained a weak independent association with $\operatorname{LASr}(R=-0,220 ; p=0,017$ and $R$ $=0,199 ; p=0,046$, respectively). In conclusion, LASr is reduced in patients with MFS, which may represent an early stage of LV diastolic dysfunction. LASr is not determined by the elastic properties of the aorta, suggesting that impaired myocardial relaxation is a primary condition in MFS.

\section{Introduction}

Marfan syndrome (MFS) is the most frequent heritable connective tissue disorder and is essentially caused by mutations in the FBN1 gene [1]. Besides abnormalities in the musculoskeletal and ocular systems, the cardiovascular manifestations represent the main clinical focus while managing MFS patients, since progressive dilatation of the aorta and acute aortic dissection are the leading causes of premature death in untreated patients [2]. In addition, the existence of primary myocardial involvement (in the absence of significant aortic or mitral regurgitation) has long been suspected in MFS patients [3]. However, the initial evidence was controversial $[4,5]$, and it was not until recent years that advances in cardiac imaging have allowed the detection of subtle cardiac impairment in this population [6-9]. Related to this, impaired myocardial relaxation has been identified in patients with MFS using conventional echocardiography $[7,10-$ 12].

Speckle-tracking echocardiography (STE) enables objective measurement of left atrial strain (LAS), which is a sensitive marker of diastolic dysfunction, showing good correlation with invasive measurements of left ventricular (LV) relaxation [13,14]. To date, there are no studies describing LAS in MFS patients. Furthermore, increased aortic wall stiffness have been documented in MFS patients [15], which may be responsible for subsequent impaired myocardial relaxation due to increased afterload.

In the present study, our aim was to characterize LV myocardial performance in a cohort of MFS patients using STE-derived measurements along with traditional echocardiographic parameters and compare them with 
healthy controls. Additionally, we sought to investigate the possible relationship between LAS and predictors of diastolic dysfunction, with a special focus on the elastic properties of the aorta.

\section{Methods}

\section{Study population:}

We consecutively included 127 MFS adult patients with normal LV ejection fraction and no previous cardiovascular surgery or significant valvular regurgitation. Marfan syndrome was diagnosed according to the Ghent criteria. We also studied 38 healthy controls (age and sex-matched).

\section{Echocardiographic evaluation:}

A comprehensive transthoracic echocardiographic study was performed using standard views.

Echocardiographic studies were performed with an iE33 ultrasound system (Philips Medical Systems, Best, the Netherlands) using an S5-1 transducer. Images were digitally stored for subsequent offline analysis. All participants had their blood pressure measured by cuff sphygmomanometer at the time of echocardiographic evaluation.

\section{Two-dimensional and Doppler quantification:}

Diastolic function evaluation and cardiac chamber quantification was performed according to latest guidelines [16,17]. Diastolic measurements included peak early and late diastolic mitral flow velocities and their ratio $\left(E, A\right.$, and $E / A$, respectively); early diastolic mitral annular velocity ( $\left.e^{\prime}\right)$ both septal and lateral, the $E / e^{\prime}$ ratio (using average of septal and lateral e'); and peak tricuspid regurgitation (TR) velocity. LV and LA volumes were obtained by Simpson's biplane method and were indexed to body surface area (BSA).

Mitral valve prolapse (MVP) was diagnosed when the systolic displacement of at least one of the mitral leaflets into the LA was $\geq 2 \mathrm{~mm}$ from the mitral annular plane in the parasternal long-axis window [18].

The aortic root diameters were carefully measured at the level of the sinuses of Valsalva perpendicular to the long axis of the aorta, using the leading-edge to leading-edge technique, following current recommendations [19]. The end-diastolic aortic diameter was considered to calculate the aortic root z-score according to Devereux's method [20], which provides a measurement of this structure corrected by sex, age and BSA.

\section{Speckle tracking echocardiography:}

Strain analysis was performed offline using 2D speckle-tracking software (QLAB 10, Philips) and following latest recommendations [21,22]. A single echocardiography specialist, who was blinded to clinical data, selected suitable echocardiographic images and performed LV and LA strain analysis. Patients were excluded 
from this analysis if none of the recorded images were of an acceptable quality. Acquisition frame rates ranged from 40 to 80 frames/s. We used the onset of the QRS as the reference point (RR gating). Adequate tracking of the endocardial LV border and LA wall was verified and if necessary adjusted to ensure reliable speckle correlation. LV global longitudinal strain (LVGLS) was calculated as the average value of the peak systolic strain curves in a 16-segment model (expressed in absolute numbers). For LAS analysis (Fig.1), we used on a non-foreshortened apical 4-chamber view. The software divided the LA wall into 7 segments, and generated longitudinal curves for each segment and a mean curve of all segments. LAS during reservoir phase (LASr) was defined as the maximum positive mean strain value during LV systole. LAS during contraction phase (LASct) was defined as the difference between the end of diastole and the onset of atrial filling (expressed in absolute numbers). In case of uncertainty when defining these phases, the mitral inflow profile was consulted for comparison. LAS during conduit phase (LAScd) was calculated as the difference between LASr and LASct (expressed also in absolute numbers).

\section{Evaluation of mechanical properties of the aorta:}

The elastic properties of the aorta were assessed by the calculation of aortic distensibility (AoD) and the stiffness index $(\mathrm{SI})$. AoD refers to relative diameter change for a pressure increment and was calculated using the following formula:

$\mathrm{AoD}=[(\mathrm{Ds}-\mathrm{Dd}) / \mathrm{Dd}] * 1 /(\mathrm{SBP}-\mathrm{DBP})$

where Ds and Dd are end-systolic and end-diastolic aortic diameters respectively (both measured at the sinuses of Valsalva), and SBP and DBP are systolic and diastolic blood pressure, respectively. SI refers to the ratio of logarithm (systolic/diastolic pressures) to relative change in diameter and was calculated using the following formula:

$\mathrm{SI}=\ln [(\mathrm{SBP} / \mathrm{DBP}) /[(\mathrm{Ds}-\mathrm{Dd}) / \mathrm{Dd}]]$

\section{Statistical analysis:}

Continuous variables were expressed as mean \pm standard deviation (SD). Categorical variables were expressed as number and percentage. The independent samples T-Test and the chi-square test were used to compare continuous and categorical variables, respectively. An analysis of which parameters determine LASr was performed, with special focus on the mechanical properties of the aorta. The strength of linear associations between LASr and possible determinants was assessed by Pearson's correlation coefficient. In addition, univariate and multivariate regression analyses were performed to assess which of these parameters were independent determinants of LASr in MFS patients.

The inter- and intra-observer variabilities were assessed for LASr in 15 healthy controls and 15 MFS patients who were randomly selected. For intra-observer variability, the same operator performed a second measurement, more than a month after the first analysis. For inter-observer variability, a second operator 
analyzed the same loops. Inter-observer and intra-observer reproducibility were assessed by calculating intraclass correlation coefficients.

IBM SPSS Statistics software (version 21.0) was used to perform statistical analysis. The results were considered statistically significant when the $p$ value was less than 0.05 .

\section{Results}

\section{Study population characteristics}

The demographic, clinical and echocardiographic characteristics of MFS patients and healthy controls are shown in Table 1. Among MFS patients, the reported use of beta-blockers and angiotensin II receptor blockers (ARBs) was $28 \%$ and $39 \%$, respectively. Measured pulse pressure was lower in the MFS sample, due to significantly lower values of SBP. The aortic root diameter and calculated SI were higher in MFS, as opposed to AoD, that was found to be reduced in this group of patients compared to controls.

We identified MVP in 61 (48\%) of all MFS patients enrolled in our study. All these cases were mild, without significant (moderate or severe) mitral regurgitation.

The MFS group exhibited increased end-diastolic and end-systolic LV volumes compared to controls (adjusted for BSA). A trend towards lower LVEF was seen in MFS patients, but still within normal limits.

According to current guidelines, it is important to mention that all participants had normal LV diastolic function. MFS patients showed lower e' velocities (septal and lateral) and higher average E/e' ratio compared to controls, but still within normal range. The analysis of mitral inflow and LA size in MFS patients revealed a slightly reduced E/A ratio and a modestly larger LAVi than healthy controls, but this difference did not reach statistical significance.

\section{Speckle-tracking analysis:}

LV strain analysis was feasible in 119 MFS patients (93.7\%) and in all healthy controls. LVGLS was significantly reduced in MFS patients, with mean values slightly below the range of normality ( 20\%). LAS analysis was feasible in 114 MFS patients $(89.8 \%)$ and in 35 healthy controls $(92.1 \%)$. We found that LASr was significantly lower in the MFS group, reflecting an impaired LV relaxation. For this reason, despite LASct was not different between the two groups, conduit function (assessed by LAScd) was also reduced in MFS patients compared to healthy controls.

\section{Reproducibility}

Inter- and intra-observer variability for LASr were assessed by the Bland-Altman method (Figure 2). The reproducibility of LASr measurements was excellent. The intraclass correlation coefficients were 0.94 (95\% Cl: $0.87-0.97)$ and 0.97 (95\% Cl: $0.95-0.99)$ for inter- and intra-observer variability, respectively. 


\section{Determinants of LASr in MFS patients}

We found no differences in LASr values regarding gender, use of beta-blockers, use of ARBs or presence of MVP (Figure 3). Therefore, we focused the analysis of possible determinants of LASr on the continuous variables. We observed weak positive correlations between LASr and some of these variables: LVEF, LVGLS, AoD, E wave, E/A ratio and e' velocities (both septal and lateral). Besides, LVESVi and aortic SI showed significant correlation with LASr, that was also weak but negative in both cases (Table 2 and Figure 4). We performed linear regression analysis including all the variables that demonstrated significant correlation with LASr (Table 3). After multivariate analysis, only LVESVi and E/A ratio remained significantly associated with LASr in MFS patients. However, the absolute value of the standardized $\beta$ coefficient was low for both variables, meaning that these variables might not be strong determinants of the reservoir function of the LA.

\section{Discussion}

Our results demonstrated that

- MFS patients, with no previous cardiovascular surgery or significant valvular regurgitation, showed a subtle impairment in certain parameters of LV myocardial performance compared to controls, including a slightly reduced LVGLS;

- despite this, the mean values of all conventional diastolic measurements were within normal limits and, more importantly, none of the participants met the criteria for abnormal diastolic function according to current guidelines;

- LAS analysis, as part of the evaluation of diastolic function, was highly feasible and reproducible in MFS patients;

- a significant reduction in LASr was observed in MFS patients compared to healthy controls, reflecting an early impairment in myocardial relaxation;

- although weak correlations were found between LASr and certain echocardiographic parameters, multivariate analysis revealed that neither AoD nor SI were independent determinants of LASr in MFS.

From a pathophysiological point of view, the myocardial dysfunction seen in MFS can be explained by the existence of abnormalities in the underlying connective tissue that results in a stiff extracellular matrix with a weakened elastic recoil [23]. Supporting this hypothesis, a number of studies have confirmed the presence of subclinical diastolic abnormalities in the MFS population. In a small observational study, De Backer et al reported a subtle diastolic impairment in MFS patients, characterized by increased deceleration time of the $\mathrm{E}$ wave and reduced septal TDI velocity [11]. Rybczynski et al found reduced early diastolic TDI velocities in a cohort of MFS adults with and without aortic root surgery [12]. Later studies reproduced similar findings $[7,10,24]$, which are also in agreement with our results. 
To the best of our knowledge, our study is the first to describe LAS analysis by STE in patients diagnosed with MFS. In a previous study, Kiotsekoglou et al performed a laborious assessment of LA function using volume measurements by Simpson method, demonstrating a reduction in reservoir, conduit and contractile function in MFS patients [10]. Our results are comparable, except we did not find any differences regarding the values of LA strain during contraction phase (LASct). In our opinion, this should be interpreted as a sign of incipient diastolic dysfunction, where early passive filling of the LV is slightly reduced while the active emptying of the LA is still preserved.

LAS has recently emerged as a promising tool in the evaluation of diastolic function. Strain analysis by STE has the added benefits of being semi-automated and angle-independent. Feasibility and reproducibility of this technique are high, as shown in our study. Remarkably, none of the individuals in the MFS cohort met the criteria for abnormal diastolic function according to current guidelines. Despite this, we found mean values of LAS among MFS patients that were clearly below the described threshold for normality $(39 \%, 23 \%$ and $18 \%$ for LASr, LAScd and LASct, respectively) [25]. Among the different measurements described in LAS analysis, we believe that LASr is the most representative parameter for various reasons. First, reduced LASr is an early marker of LV stiffness that reflects the compromised ability of the atrium to act as a reservoir during ventricular systole, thereby arising before structural remodeling [26]. Second, LASr has an excellent agreement with invasively determined pressures - especially in patients with normal LVEF [27] - and allows, as a single marker, an accurate categorization of the degree of diastolic dysfunction [28]. Finally, decreased LASr has been linked to higher risk of cardiovascular events in different clinical scenarios [29-32]. For this reason, we believe that our results are of great relevance and might set the basis for future investigations exploring the prognostic value of LASr in MFS patients.

In the literature, the reported prevalence of MVP in MFS is widely variable. The proportion of at least mild mitral valve pathology has been estimated to be approximately $75 \%$, whereas the prevalence of more severe myxomatous thickening with prolapse is closer to $25 \%$ in these individuals [33]. In our MFS cohort, almost half of the patients had MVP. Neither of these patients had significant mitral regurgitation. Noteworthy, the value of LASr was similar between individuals with and without MVP, meaning that MVP alone is not associated with functional impairment of the LA.

There is limited evidence about the possible natural determinants of LA strain. Recent studies have suggested that age, LV strain, average TDI e' velocity and LAVi may be predictors of LASr [34-36]. In our study, LVESVi and $E / A$ ratio were the only independent determinants of LASr. These correlations were weak, but our results agree with the existence of an intimate link between LASr and LV mechanics. 
As mentioned above, reduced LA strain is a sign of poor LA compliance. This situation could also be a consequence of atrial structural remodeling due to increased interstitial fibrosis, usually accompanied by LA enlargement. Conversely, atrial functional remodeling results in decreased function with or without changes in LA size [37]. In our study, LAVi was comparable in MFS and healthy controls. Additionally, we did not find a significant correlation between LASr and LAVi in MFS patients. Thus, based on our results, we consider that impaired LASr was most likely due to impaired LV relaxation rather than intrinsic remodeling of the LA.

None of the elastic properties of the aorta (AoD or SI) showed independent associations with LASr in our MFS cohort. Univariate linear regression did find a weak correlation between these variables and reservoir strain. However, after adjustment for parameters related to myocardial function, this association lost significance. Similar findings have been reported by others [8,38], observing abnormalities in LV function that were independent from measurable derivatives of aortic elasticity. In this context, it should be mentioned that altered diastolic function has been described already in children with MFS [39,40]. Such findings in the early childhood, along with our results, further support the hypothesis that impaired myocardial relaxation may be a primary condition rather than a consequence of reduced aortic compliance in patients with MFS.

\section{Limitations}

A number of limitations should be considered. First, our study was conducted in a single center, which implies that our results are difficult to generalize. Second, the strain analysis was performed using a single-vendor software, which also limits the external reproducibility of our results. Furthermore, this software was not specifically designed for LAS analysis. However, the application of LV software packages for the study of LA function has been widely used and validated in clinical practice. Third, phenotypic-genotypic correlation was missing in our study. Despite a substantial proportion of the enrolled individuals had genetic testing performed, there was a large spectrum of different mutations that hindered the search for interpretable associations between genotype and myocardial abnormalities.

\section{Conclusions}

This study demonstrates that patients with MFS, in the absence of previous cardiac surgery or significant valvular regurgitation, present with reduced values of LASr as a marker of subclinical diastolic dysfunction. According to our results, reduced LASr is not determined by the elastic properties of the aorta, supporting the hypothesis that impaired myocardial relaxation is a primary condition in MFS. Future investigations should address the prognostic value of LAS in these patients, as well as the role of preventive and therapeutic interventions in the setting of MFS-related cardiomyopathy.

\section{Declarations}

\section{SOURCES OF FUNDING}


This work was supported by a grant from Comunidad de Madrid (AORTASANA-CM; B2017/BMD-3676).

\section{CONFLICTS OF INTEREST}

None

\section{ETHICAL APPROVAL}

Ethics approval was obtained from the Research Ethics Committee of Puerta de Hierro University Hospital in Madrid (Spain). Each participant provided written informed consent after receiving a detailed description of the study

\section{References}

1. Robinson PN, Arteaga-Solis E, Baldock C, Collod-Béroud G, Booms P, De Paepe A, Dietz HC, Guo G, Handford PA, Judge DP et al. The molecular genetics of Marfan syndrome and related disorders. J Med Genet. 2006;43:769-787. doi: 10.1136/jmg.2005.039669

2. Judge DP, Dietz HC. Marfan's syndrome. Lancet. 2005;366:1965-1976. doi: 10.1016/S01406736(05)67789-6

3. Whitfield AGW, Arnott WM, Stafford JL. 'Myocarditis' and aortic hypoplasia in arachnodactyly: report of a case. Lancet. 1951;257:1387-1391. doi: 10.1016/s0140-6736(51)92841-3

4. Chatrath R, Beauchesne LM, Connolly HM, Michels VV, Driscoll DJ. Left ventricular function in the Marfan syndrome without significant valvular regurgitation. Am J Cardiol. 2003;91:914-916. doi: 10.1016/s00029149(03)00039-0

5. Meijboom LJ, Timmermans J, Tintelen JP van, Nollen GJ, De Backer J, Berg MP van den, Boers GH, Mulder BJM. Evaluation of left ventricular dimensions and function in Marfan's syndrome without significant valvular regurgitation. Am J Cardiol. 2005;95:795-797. doi: 10.1016/j.amjcard.2004.11.042

6. Alpendurada F, Wong J, Kiotsekoglou A, Banya W, Child A, Prasad SK, Pennell DJ, Mohiaddin RH. Evidence for Marfan cardiomyopathy. Eur J Heart Fail. 2010;12:1085-1091. doi: 10.1093/eurjhf/hfq127

7. Scherptong RWC, Vliegen HW, Wall EE van der, Hilhorst-Hofstee Y, Bax JJ, Scholte AJ, Delgado V. Biventricular performance in patients with marfan syndrome without significant valvular disease: comparison to normal subjects and longitudinal follow-up. J Am Soc Echocardiogr. 2011;24:13921399.e1. doi: 10.1016/j.echo.2011.09.004

8. Witte P de, Aalberts JJJ, Radonic T, Timmermans J, Scholte AJ, Zwinderman AH, Mulder BJM, Groenink M, Berg MP van den. Intrinsic biventricular dysfunction in Marfan syndrome. Heart. 2011;97:2063-2068. doi: 10.1136/heartjnl-2011-300169

9. Winther S, Williams LK, Keir M, Connelly KA, Bradley TJ, Rakowski H, Crean AM. Cardiovascular Magnetic Resonance Provides Evidence of Abnormal Myocardial Strain and Primary Cardiomyopathy in Marfan syndrome. J Comput Assist Tomogr. 2019;43(3):410-415. doi: 10.1097/RCT.0000000000000863.

10. Kiotsekoglou A, Moggridge JC, Bijnens BH, Kapetanakis V, Alpendurada F, Mullen MJ, Saha S, Nassiri DK, Camm J, Sutherland GR, Child AH. Biventricular and atrial diastolic function assessment using 
conventional echocardiography and tissue-Doppler imaging in adults with Marfan syndrome. Eur $\mathrm{J}$ Echocardiogr. 2009;10:947-955. doi: 10.1093/ejechocard/jep110

11. De Backer JF, Devos D, Segers P, Matthys D, François K, Gillebert TC, De Paepe AM, De Sutter J. Primary impairment of left ventricular function in Marfan syndrome. Int J Cardiol. 2006;112:353-358. doi: 10.1016/j.ijcard.2005.10.010

12. Rybczynski M, Koschyk DH, Aydin MA, Robinson PN, Brinken T, Franzen O, Berger J, Hofmann T, Meinertz T, Kodolitsch $Y$ von. Tissue Doppler imaging identifies myocardial dysfunction in adults with Marfan syndrome. Clin Cardiol. 2007;30:19-24. doi: 10.1002/clc.3

13. Cameli M, Mandoli GE, Loiacono F, Dini FL, Henein M, Mondillo S. Left atrial strain: a new parameter for assessment of left ventricular filling pressure. Heart Fail Rev. 2016;21:65-76. doi: 10.1007/s10741-0159520-9

14. Buggey J, Hoit BD. Left atrial strain: measurement and clinical application. Curr Opin Cardiol. 2018;33:479-485. doi: 10.1097/HC0.0000000000000537

15. Salvi P, Grillo A, Marelli S, Gao L, Salvi L, Viecca M, Di Blasio AM, Carretta R, Pini A, Parati G. Aortic dilatation in Marfan syndrome: role of arterial stiffness and fibrillin-1 variants. J Hypertens. 2018;36:7784. doi: $10.1097 / \mathrm{HJH} .0000000000001512$

16. Lang RM, Badano LP, Mor-Avi V, Afilalo J, Armstrong A, Ernande L, Flachskampf FA, Foster E, Goldstein SA, Kuznetsova $T$ et al. Recommendations for cardiac chamber quantification by echocardiography in adults: an update from the American Society of Echocardiography and the European Association of Cardiovascular Imaging. J Am Soc Echocardiogr. 2015;28:1-39.e14. doi: 10.1016/j.echo.2014.10.003.

17. Nagueh SF, Smiseth OA, Appleton CP, Byrd BF, Dokainish H, Edvardsen T, Flachskampf FA, Gillebert TC, Klein AL, Lancellotti P et al. Recommendations for the Evaluation of Left Ventricular Diastolic Function by Echocardiography: An Update from the American Society of Echocardiography and the European Association of Cardiovascular Imaging. J Am Soc Echocardiogr. 2016;29:277-314. doi: 10.1016/j.echo.2016.01.011.

18. Zoghbi WA, Adams D, Bonow RO, Enriquez-Sarano M, Foster E, Grayburn PA, Hahn RT, Han Y, Hung J, Lang RM et al. Recommendations for Noninvasive Evaluation of Native Valvular Regurgitation: A Report from the American Society of Echocardiography Developed in Collaboration with the Society for Cardiovascular Magnetic Resonance. J Am Soc Echocardiogr. 2017;30:303-371. doi: 10.1016/j.echo.2017.01.007

19. Goldstein SA, Evangelista A, Abbara S, Arai A, Asch FM, Badano LP, Bolen MA, Connolly HM, CuéllarCalàbria $\mathrm{H}$, Czerny $\mathrm{M}$ et al. Multimodality imaging of diseases of the thoracic aorta in adults: from the American Society of Echocardiography and the European Association of Cardiovascular Imaging: endorsed by the Society of Cardiovascular Computed Tomography and Society for Cardiovascular Magnetic Resonance. J Am Soc Echocardiogr. 2015;28:119-182. doi: 10.1016/j.echo.2014.11.015

20. Devereux RB, Simone G de, Arnett DK, Best LG, Boerwinkle E, Howard BV, Kitzman D, Lee ET, Mosley TH, Weder A, Roman MJ. Normal limits in relation to age, body size and gender of two-dimensional echocardiographic aortic root dimensions in persons $\geq 15$ years of age. Am J Cardiol. 2012;110:11891194. doi: 10.1016/j.amjcard.2012.05.063

21. Badano LP, Kolias TJ, Muraru D, Abraham TP, Aurigemma G, Edvardsen T, D'Hooge J, Donal E, Fraser AG, Marwick $T$ et al. Standardization of left atrial, right ventricular, and right atrial deformation imaging using 
two-dimensional speckle tracking echocardiography: a consensus document of the EACVI/ASE/Industry Task Force to standardize deformation imaging. Eur Heart J Cardiovasc Imaging. 2018;19:591-600. doi: 10.1093/ehjci/jey042

22. Voigt JU, Pedrizzetti G, Lysyansky P, Marwick TH, Houle H, Baumann R, Pedri S, Ito Y, Abe Y, Metz S et al. Definitions for a common standard for 2D speckle tracking echocardiography: consensus document of the EACVI/ASE/Industry Task Force to standardize deformation imaging. Eur Heart J Cardiovasc Imaging. 2015 Jan;16(1):1-11. doi: 10.1093/ehjci/jeu184.

23. Demolder A, Kodolitsch Y von, Muiño-Mosquera L, De Backer J. Myocardial Function, Heart Failure and Arrhythmia in Marfan Syndrome: A Systematic Literature Review. Diagnostics (Basel). 2020;10:751. doi: 10.3390/diagnostics10100751

24. Kiotsekoglou A, Saha S, Moggridge JC, Kapetanakis V, Govindan M, Alpendurada F, Mullen MJ, Nassiri DK, Camm J, Sutherland GR et al. Impaired biventricular deformation in Marfan syndrome: a strain and strain rate study in adult unoperated patients. Echocardiography. 2011;28:416-430. doi: 10.1111/j.15408175.2010.01359.x

25. Thomas L, Muraru D, Popescu BA, Sitges M, Rosca M, Pedrizzetti G, Henein MY, Donal E, Badano LP. Evaluation of Left Atrial Size and Function: Relevance for Clinical Practice. J Am Soc Echocardiogr. 2020;33:934-952. doi: 10.1016/j.echo.2020.03.021

26. Morris DA, Takeuchi M, Krisper M, Köhncke C, Bekfani T, Carstensen T, Hassfeld S, Dorenkamp M, Otani K, Takigiku $\mathrm{K}$ et al. Normal values and clinical relevance of left atrial myocardial function analysed by speckle-tracking echocardiography: multicentre study. Eur Heart J Cardiovasc Imaging. 2015;16:364-372. doi: 10.1093/ehjci/jeu219

27. Singh A, Medvedofsky D, Mediratta A, Balaney B, Kruse E, Ciszek B, Shah AP, Blair JE, Maffessanti F, Addetia $\mathrm{K}$ et al. Peak left atrial strain as a single measure for the non-invasive assessment of left ventricular filling pressures. Int J Cardiovasc Imaging. 2019;35:23-32. doi: 10.1007/s10554-018-1425-y

28. Singh A, Addetia K, Maffessanti F, Mor-Avi V, Lang RM. LA Strain for Categorization of LV Diastolic Dysfunction. JACC Cardiovasc Imaging. 2017;10:735-743. doi: 10.1016/j.jcmg.2016.08.014

29. Cameli M, Lisi M, Focardi M, Reccia R, Natali BM, Sparla S, Mondillo S. Left Atrial Deformation Analysis by Speckle Tracking Echocardiography for Prediction of Cardiovascular Outcomes. Am J Cardiol. 2012;110:264-269. doi: 10.1016/j.amjcard.2012.03.022

30. Ersbøll M, Andersen MJ, Valeur N, Mogensen UM, Waziri H, Møller JE, Hassager C, Søgaard P, Køber L. The Prognostic Value of Left Atrial Peak Reservoir Strain in Acute Myocardial Infarction Is Dependent on Left Ventricular Longitudinal Function and Left Atrial Size. Circ Cardiovasc Imaging. 2013;6:26-33. doi: 10.1161/CIRCIMAGING.112.978296

31. Santos ABS, Roca GQ, Claggett B, Sweitzer NK, Shah SJ, Anand IS, Fang JC, Zile MR, Pitt B, Solomon SD, Shah AM. The Prognostic Relevance of Left Atrial Dysfunction in Heart Failure With Preserved Ejection Fraction. Circ Heart Fail. 2016;9:e002763. doi: 10.1161/CIRCHEARTFAILURE.115.002763

32. Kim J, Yum B, Palumbo MC, Sultana R, Wright N, Das M, You C, Moskowitz CS, Levine RA, Devereux RB, Weinsaft JW. Left Atrial Strain Impairment Precedes Geometric Remodeling as a Marker of PostMyocardial Infarction Diastolic Dysfunction. JACC Cardiovasc Imaging. 2020;13:2099-2113. doi: 10.1016/j.jcmg.2020.05.041 
33. Delling FN, Vasan RS. Epidemiology and Pathophysiology of Mitral Valve Prolapse: New Insights into Disease Progression, Genetics, and Molecular Basis. Circulation. 2014;129:2158-2170. doi: 10.1161/CIRCULATIONAHA.113.006702

34. Gan GCH, Bhat A, Chen HHL, Fernandez F, Byth K, Eshoo S, Thomas L. Determinants of LA reservoir strain: Independent effects of LA volume and LV global longitudinal strain. Echocardiography. Online ahead of print. doi: 10.1111/echo.14922

35. Sun BJ, Park J-H, Lee M, Choi J-O, Lee J-H, Shin M-S, Kim M-J, Jung HO, Park JR, Sohn IS et al. Normal Reference Values for Left Atrial Strain and Its Determinants from a Large Korean Multicenter Registry. J Cardiovasc Imaging. 2020;28:186-198. doi: 10.4250/jcvi.2020.0043

36. Inoue K, Khan FH, Remme EW, Ohte N, García-Izquierdo E, Chetrit M, Moñivas-Palomero V, Mingo-Santos S, Andersen ØS, Gude E et al. Determinants of left atrial reservoir and pump strain and use of atrial strain for evaluation of left ventricular filling pressure. Eur Heart J Cardiovasc Imaging. 2021 Jan 26:jeaa415. doi: 10.1093/ehjci/jeaa415.

37. Thomas L, Abhayaratna WP. Left Atrial Reverse Remodeling: Mechanisms, Evaluation, and Clinical Significance. JACC Cardiovasc Imaging. 2017;10:65-77. doi: 10.1016/j.jcmg.2016.11.003

38. Loeper F, Oosterh of J, Dorpel M van den, Linde D van der, Lu Y, Robertson E, Hambly B, Jeremy R. Ventricular-Vascular Coupling in Marfan and Non-Marfan Aortopathies. J Am Heart Assoc. 2016;5:e003705. doi: 10.1161/JAHA.116.003705

39. Savolainen A, Nisula L, Keto P, Hekali P, Viitasalo M, Kaitila I, Kupari M. Left ventricular function in children with the Marfan syndrome. Eur Heart J. 1994;15:625-630. doi: 10.1093/oxfordjournals.eurheartj.a060558

40. Das BB, Taylor AL, Yetman AT. Left ventricular diastolic dysfunction in children and young adults with Marfan syndrome. Pediatr Cardiol. 2006;27:256-258. doi: 10.1007/s00246-005-1139-5

\section{Tables}

\section{Table 1. Baseline characteristics}




\begin{tabular}{|c|c|c|c|}
\hline & MFS patients $(n=127)$ & Controls $(n=38)$ & $\mathrm{p}$ \\
\hline Age & $33.5 \pm 13.0$ & $32.5 \pm 8.1$ & 0.542 \\
\hline Male (\%) & $61(48 \%)$ & $18(47 \%)$ & 0.943 \\
\hline Height $(\mathrm{cm})$ & $182.9 \pm 11.1$ & $171.0 \pm 10.4$ & $<0.001$ \\
\hline Weigth (kg) & $74.1 \pm 16.5$ & $66.6 \pm 9.9$ & 0.001 \\
\hline $\mathrm{BSA}\left(\mathrm{m}^{2}\right)$ & $1.95 \pm 0.25$ & $1.78 \pm 0.18$ & $<0.001$ \\
\hline $\mathrm{SBP}(\mathrm{mmHg})$ & $118.5 \pm 12.7$ & $123.8 \pm 9.8$ & 0.010 \\
\hline $\mathrm{DBP}(\mathrm{mmHg})$ & $71.0 \pm 10.0$ & $71.2 \pm 9.4$ & 0.915 \\
\hline Pulse pressure (mmHg) & $47.5 \pm 9.9$ & $52.6 \pm 9.6$ & 0.007 \\
\hline Aortic root $(\mathrm{mm})$ & $39.1 \pm 5.3$ & $30.1 \pm 3.0$ & $<0.001$ \\
\hline Z-Score & $2.54 \pm 1.39$ & $0.13 \pm 0.93$ & $<0.001$ \\
\hline Aortic distensibility $(\mathrm{mm} \mathrm{Hg}-1) \times 10^{-3}$ & $0.97 \pm 0.45$ & $1.55 \pm 0.56$ & $<0.001$ \\
\hline Stiffness index & $3.72 \pm 0.45$ & $3.15 \pm 0.36$ & $<0.001$ \\
\hline LVEDVi $\left(\mathrm{ml} / \mathrm{m}^{2}\right)$ & $52.2 \pm 10.8$ & $46.7 \pm 10.5$ & 0.006 \\
\hline LVESVi $\left(\mathrm{ml} / \mathrm{m}^{2}\right)$ & $21.4 \pm 5.7$ & $18.2 \pm 4.5$ & 0.002 \\
\hline LVEF (\%) & $59.3 \pm 5.5$ & $61.0 \pm 4.2$ & 0.068 \\
\hline E-wave $(\mathrm{cm} / \mathrm{s})$ & $75.1 \pm 15.8$ & $81.6 \pm 14.2$ & 0.025 \\
\hline A-wave $(\mathrm{cm} / \mathrm{s})$ & $54.1 \pm 13.6$ & $53.6 \pm 12.7$ & 0.848 \\
\hline E/A ratio & $1.5 \pm 0.4$ & $1.6 \pm 0.4$ & 0.084 \\
\hline Lateral e' (cm/s) & $12.6 \pm 3.5$ & $16.5 \pm 3.2$ & $<0.001$ \\
\hline Septal e' (cm/s) & $9.6 \pm 2.2$ & $12.2 \pm 2.3$ & $<0.001$ \\
\hline Average $\mathrm{E} / \mathrm{e}^{\prime}$ ratio & $7.0 \pm 1.8$ & $5.8 \pm 1.0$ & $<0.001$ \\
\hline TR velocity $(\mathrm{cm} / \mathrm{s})$ & $206.2 \pm 24.5$ & $200.5 \pm 24.9$ & 0.440 \\
\hline $\operatorname{LAVi}\left(\mathrm{ml} / \mathrm{m}^{2}\right)$ & $25.4 \pm 7.7$ & $22.8 \pm 5.4$ & 0.062 \\
\hline \multicolumn{4}{|l|}{ STE-derived measurements: } \\
\hline LVGLS (\%) & $19.3 \pm 2.6$ & $21.6 \pm 2.1$ & $<0.001$ \\
\hline LASr (\%) & $32.9 \pm 8.5$ & $43.3 \pm 7.8$ & $<0.001$ \\
\hline LASct (\%) & $13.4 \pm 6.1$ & $14.5 \pm 4.9$ & 0.327 \\
\hline LAScd (\%) & $18.9 \pm 7.2$ & $28.9 \pm 5.7$ & $<0.001$ \\
\hline
\end{tabular}


Values are expressed as mean \pm SD or $n(\%)$. BSA, body surface area; DBP, diastolic blood pressure; LASr, LAScd and LASct: left atrial strain during reservoir, conduit and contraction phase, respectively; LAVi, left atrial volume indexed to BSA; LVEDVi, left ventricular end-diastolic volume indexed to BSA; LVEF, left ventricular ejection fraction; LVESVi, left ventricular end-systolic volume indexed to BSA; LVGLS, left ventricular global longitudinal strain; SBP, systolic blood pressure; STE, speckle-tracking echocardiography; TR, tricuspid regurgitation.

Table 2. Correlations between LASr and continuous variables in MFS patients 


\begin{tabular}{|lll|}
\hline & Pearson Correlation & $p$ value \\
\hline LASr & 1.000 & \\
\hline Age & 0.095 & 0.316 \\
\hline Height & -0.066 & 0.484 \\
\hline Weight & -0.105 & 0.267 \\
\hline BSA & -0.100 & 0.289 \\
\hline SBP & 0.067 & 0.484 \\
\hline DBP & 0.021 & 0.830 \\
\hline PP & 0.064 & 0.505 \\
\hline LVEDVi & -0.133 & 0.160 \\
\hline LVESVi & -0.210 & $0.026^{\star}$ \\
\hline LVEF & 0.214 & $0.022^{\star}$ \\
\hline LVGLS & 0.201 & $0.033^{\star}$ \\
\hline Aortic root & -0.124 & 0.189 \\
\hline Z-Score & -0.163 & 0.082 \\
\hline Aortic distensibility & 0.222 & $0.020^{\star}$ \\
\hline Stiffness index & -0.216 & $0.024^{\star}$ \\
\hline E-wave & 0.226 & $0.008^{\star}$ \\
\hline A-wave & -0.041 & 0.331 \\
\hline E/A ratio & 0.258 & $0.006^{\star}$ \\
\hline Lateral e' & 0.210 & $0.013^{\star}$ \\
\hline Septal e' & 0.185 & $0.026^{\star}$ \\
\hline Average E/e' ratio & -0.017 & 0.859 \\
\hline TR velocity & -0.022 & 0.850 \\
\hline LAVi & -0.131 & 0.165 \\
\hline
\end{tabular}

(*) Significant correlations with $\mathrm{p}$ value $<0.05$

BSA, body surface area; DBP, diastolic blood pressure; LASr, LAScd and LASct: left atrial strain during reservoir, conduit and contraction phase, respectively; LAVi, left atrial volume indexed to BSA; LVEDVi, left ventricular end-diastolic volume indexed to BSA; LVEF, left ventricular ejection fraction; LVESVi, left ventricular endsystolic volume indexed to BSA; LVGLS, left ventricular global longitudinal strain; SBP, systolic blood pressure; $T R$, tricuspid regurgitation. 
Table 3. Linear regression analysis of determinants of LA reservoir strain in MFS patients.

\begin{tabular}{|c|c|c|c|c|c|c|}
\hline & \multicolumn{3}{|c|}{ Univariate analysis } & \multicolumn{3}{|c|}{ Multivariate analysis } \\
\hline & $\begin{array}{l}\text { Unstandardized } \\
\text { coefficient } \\
(95 \% \mathrm{Cl})\end{array}$ & $\begin{array}{l}\text { Standardized } \\
\beta^{*}\end{array}$ & $\begin{array}{l}p \\
\text { value }\end{array}$ & $\begin{array}{l}\text { Unstandardized } \\
\text { coefficient } \\
(95 \% \mathrm{Cl})\end{array}$ & $\begin{array}{l}\text { Standardized } \\
\beta\end{array}$ & $\begin{array}{l}\mathrm{p} \\
\text { value }\end{array}$ \\
\hline LVESVi & $\begin{array}{l}-0.315(-0.592 \\
\text { to }-0.039)\end{array}$ & -0.210 & 0.026 & $\begin{array}{l}-0.334(-0.606 \\
\text { to }-0.061)\end{array}$ & -0.220 & 0.017 \\
\hline LVEF & $\begin{array}{l}0.339 \text { ( } 0.049 \text { to } \\
0.629)\end{array}$ & 0.214 & 0.022 & $\begin{array}{l}0.106(-0.306 \text { to } \\
0.518)\end{array}$ & 0.066 & 0.610 \\
\hline E-wave & $\begin{array}{l}0.121 \text { ( } 0.023 \text { to } \\
0.219)\end{array}$ & 0.226 & 0.016 & $\begin{array}{l}0.017(-0.126 \text { to } \\
0.160)\end{array}$ & 0.030 & 0.811 \\
\hline E/A ratio & $\begin{array}{l}4.987 \text { (1.488 to } \\
8.486)\end{array}$ & 0.258 & 0.006 & $\begin{array}{l}3.837 \text { ( } 0.065 \text { to } \\
7.608)\end{array}$ & 0.199 & 0.046 \\
\hline Lateral e' & $\begin{array}{l}0.513(0.065 \text { to } \\
0.961)\end{array}$ & 0.210 & 0.025 & $\begin{array}{l}0.269(-0.218 \text { to } \\
0.756)\end{array}$ & 0.109 & 0.275 \\
\hline Septal e' & $\begin{array}{l}0.713(-0.007 \text { to } \\
1.433)\end{array}$ & 0.185 & 0.052 & $\begin{array}{l}0.092(-1.201 \text { to } \\
1.384)\end{array}$ & 0.024 & 0.888 \\
\hline $\begin{array}{l}\text { Stiffness } \\
\text { index }\end{array}$ & $\begin{array}{l}-4.099(-7.652 \\
\text { to }-0.546)\end{array}$ & -0.216 & 0.024 & $\begin{array}{l}-0.057(-10.032 \\
\text { to } 9.917)\end{array}$ & -0.003 & 0.991 \\
\hline $\begin{array}{l}\text { Aortic } \\
\text { distensibility }\end{array}$ & $\begin{array}{l}4.201 \text { (0.665 to } \\
7.737)\end{array}$ & 0.222 & 0.020 & $\begin{array}{l}2.948(-0.549 \text { to } \\
6.445)\end{array}$ & 0.156 & 0.098 \\
\hline LVGLS & $\begin{array}{l}0.671 \text { ( } 0.054 \text { to } \\
1.289)\end{array}$ & 0.201 & 0.033 & $\begin{array}{l}0.336(-0.292 \text { to } \\
0.964)\end{array}$ & 0.101 & 0.291 \\
\hline
\end{tabular}

(*) Standardized beta coefficient equals Pearson correlation coefficient and compares the strength of the effect of each individual independent variable to the dependent variable. The higher the absolute value of the beta coefficient, the stronger the effect.

LVEF, left ventricular ejection fraction; LVESVi, left ventricular end-systolic volume indexed to BSA; LVGLS, left ventricular global longitudinal strain.

\section{Figures}




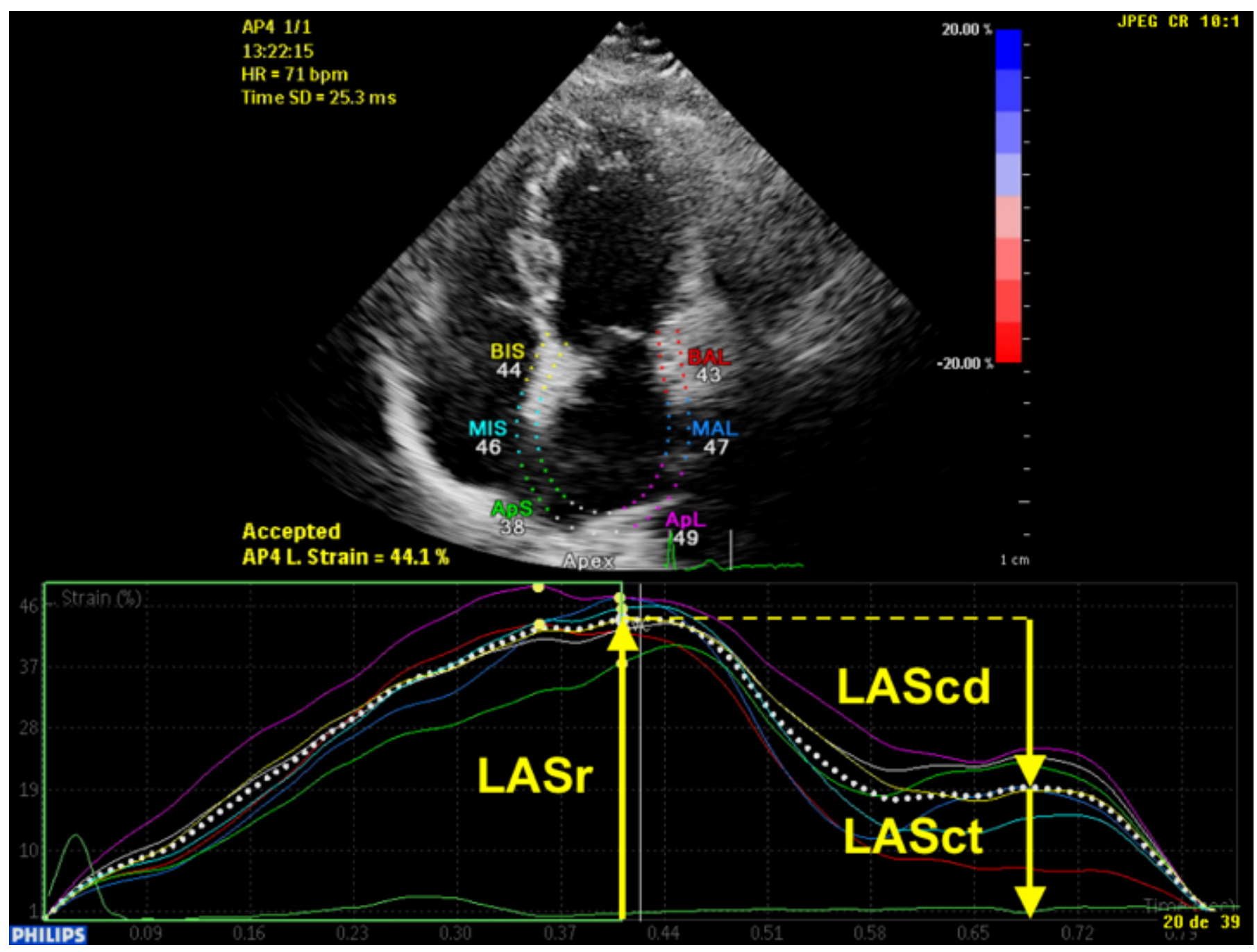

Figure 1

Example of LAS measurement (apical 4-chamber view) in a healthy control using 7 segments (different colours) with corresponding strain curves, and average strain curve (white dotted line). LASr: LA strain during reservoir phase; LAScd: LA strain during conduit phase; LASct: LA strain during contraction phase. 

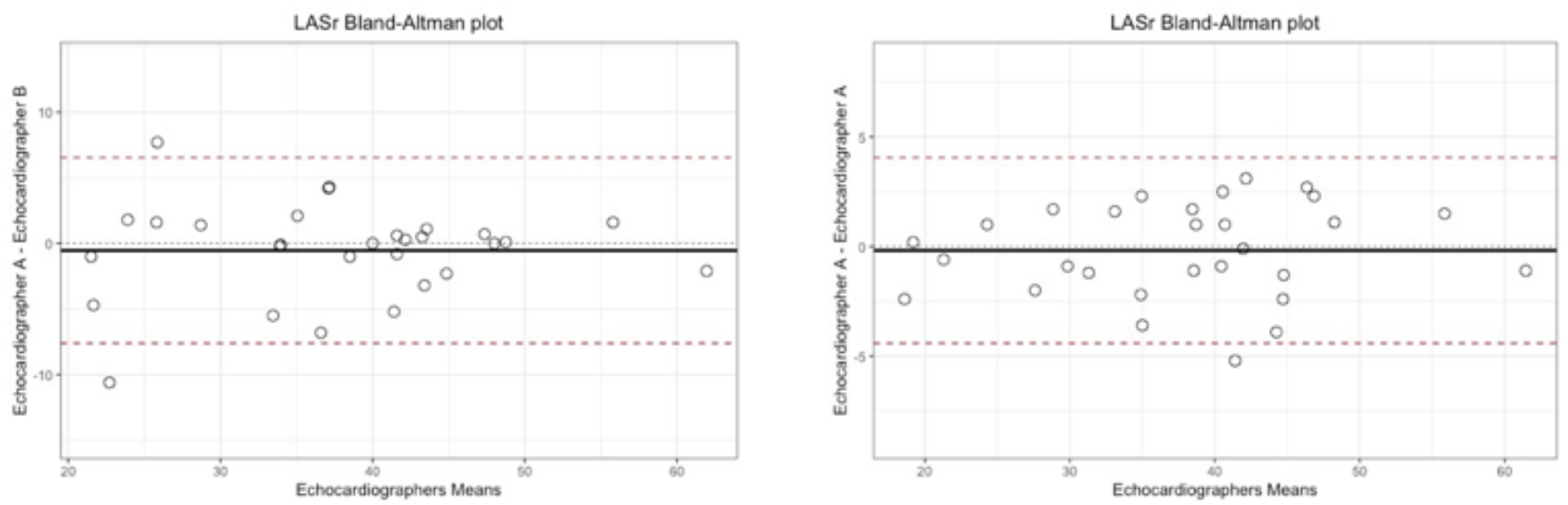

\section{Figure 2}

Inter-observer (left) and intra-observer (right) variability for LASr assessed by Bland-Altman plots. The difference in each pair of measurements was plotted against the pair's mean. 

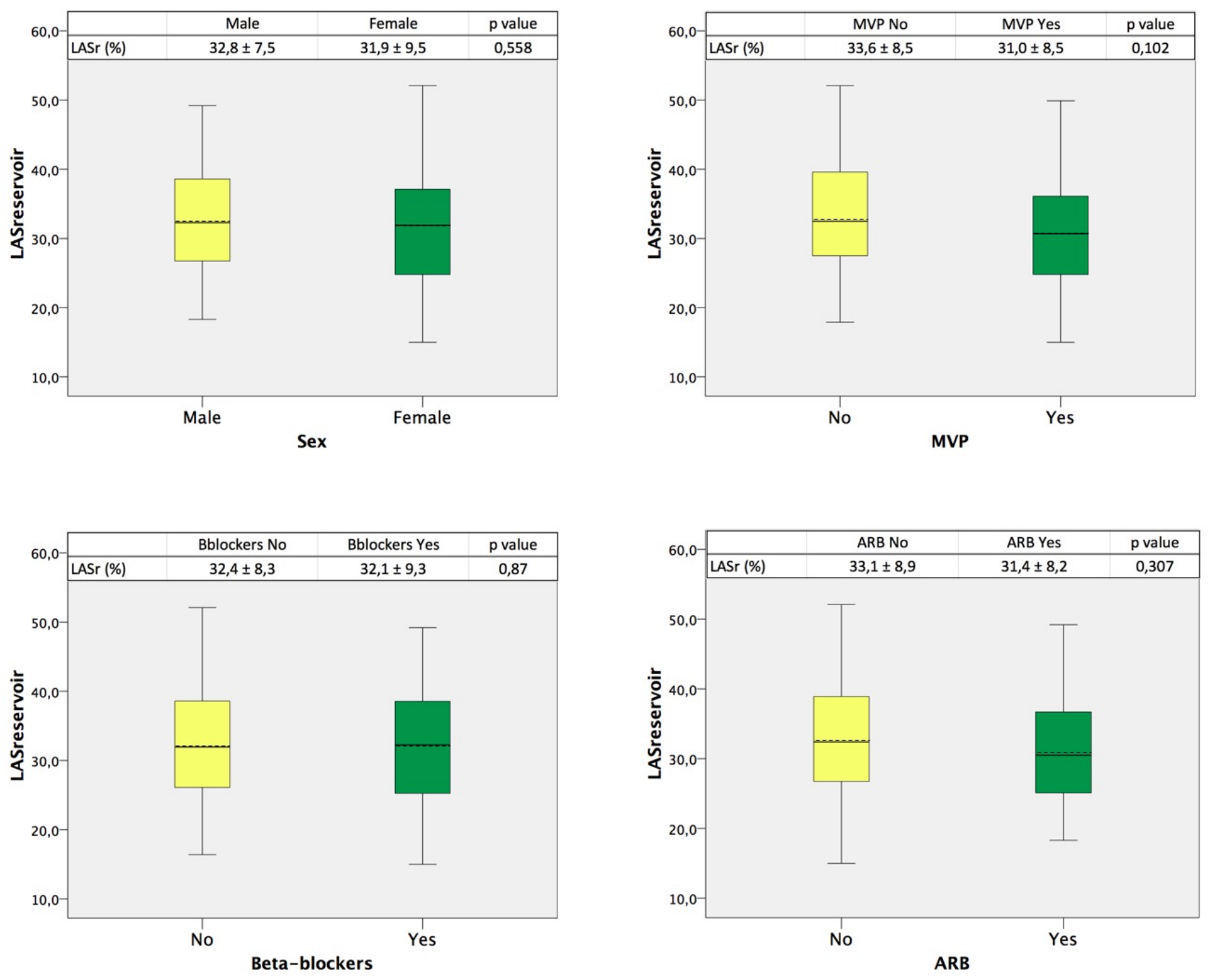

\section{Figure 3}

Comparison of LASr values according to gender, mitral valve prolapse (MVP), treatment with beta-blockers and angiotensin II receptor blockers (ARB). No significant differences were observed. Values are expressed as mean \pm SD. 

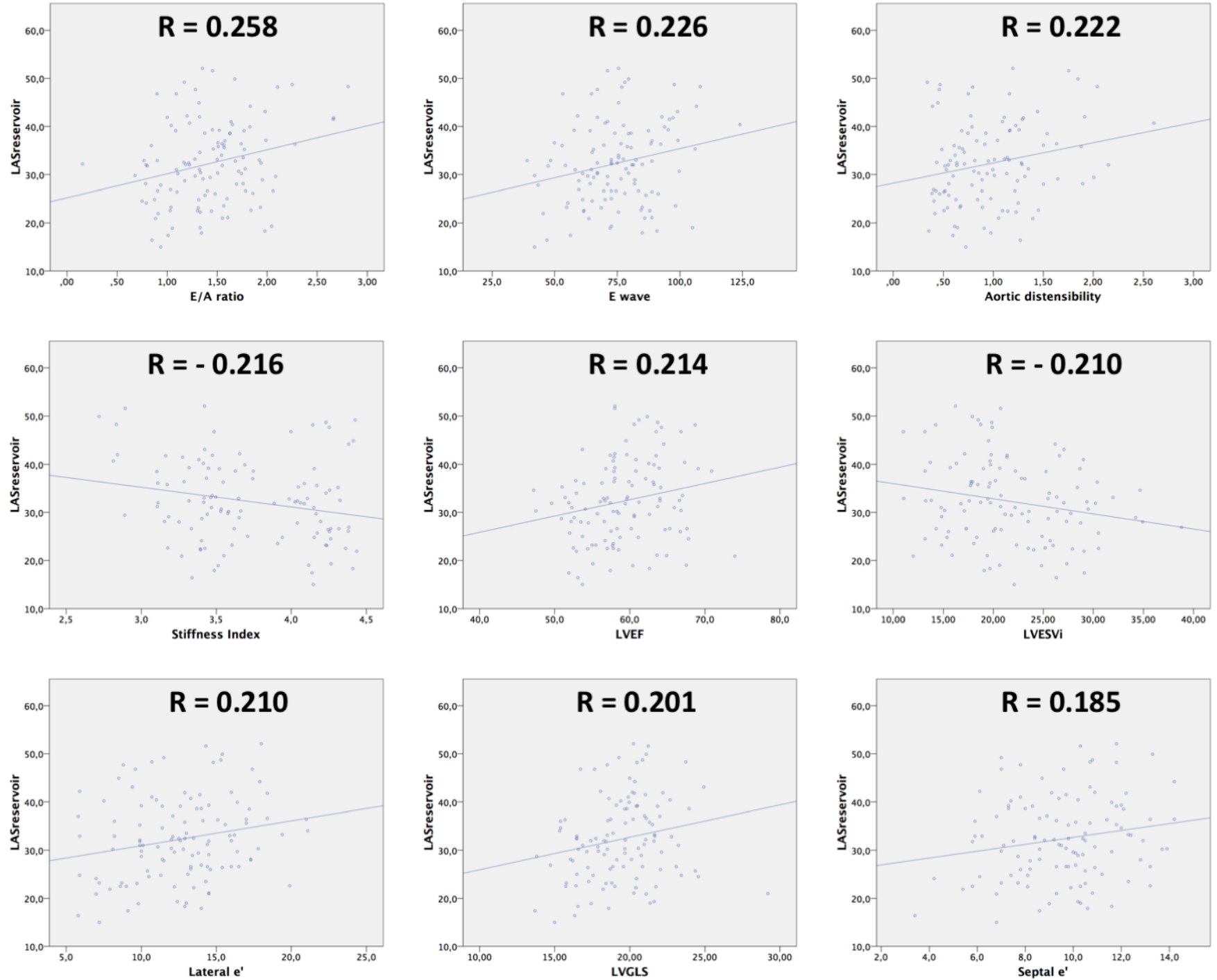

Figure 4

Regression plots demonstrating the relationship between LASr and its possible determinants. Only the continuous variables showing significant correlations $(p<0,05)$ with LASr are depicted in this figure. 